\title{
Resaerch Paper: Online Cognitive Bias Modification of Interpretation for Children With Obsessive-Compulsive Disorder
}

\author{
Saeed Nasiry ${ }^{*}$ (1), Zahra Ameli ${ }^{2}$, Pegah Pezeshki ${ }^{1}$ (]) \\ 1. Department of Clinical Psychology, School of Medicine, Shahid Beheshti University of Medical Sciences, Tehran, Iran. \\ 2. Department of Social Sciences, Faculty of Letters and Human Sciences, Shahid Beheshti University, Tehran, Iran.
}

\begin{tabular}{|c|c|}
\hline $\begin{array}{l}\text { Use vour device toscan } \\
\text { and read the article online }\end{array}$ & Citzation: Nasiry, S.. Ameli. Z., \& Pezeshki. P. (2020). Online Cognitive Bias Modification of Interpretation for Children With \\
\hline 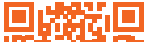 & Obsessive-Compulsive Disorder. Journal of Practice in Clinical Psychology, 8(4), 325-334. https://doi.org/10.32598/jpcp.8.4.739. \\
\hline 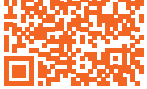 & doi https://doi.org/10.32598/jpcp.8.4.739.1 \\
\hline
\end{tabular}

\section{(i) (3)}

\section{Article info:}

Received: 20 Jan 2020

Accepted: 20 Aug 2020

Available Online: 01 Oct 2020

\section{Keywords:}

Cognitive bias modification, Interpretation bias, ObsessiveCompulsive disorder, Online intervention, Children

\section{ABSTRACT}

Objective: Numerous children with Obsessive-Compulsive Disorder (OCD) either have no access to its main treatment, i.e. Cognitive Behavioral Therapy (CBT), or fail to respond to it. Cognitive Bias Modification of Interpretation (CBMI) is a novel and promising intervention that targets the incorrect interpretation of intrusive thoughts and impulses, i.e. the characteristics of OCD. The present study aimed to determine the effects of CBMI in children with OCD for the first time. Besides, we evaluated the possibility of online implementation of this intervention.

Methods: A sample of 35 children with OCD (aged 7-12 years) were randomly assigned to two study groups. The experimental group $(n=18)$ received CBMI and the controls $(n=17)$ received placebo treatment. Interpretation bias and OCD severity were assessed at pre-test, post-test, and 2-month follow-up stages, using the Obsessive Compulsive Inventory-Child Version (OCICV), Obsessive Beliefs Questionnaire-Child Version (OBQCV), and Ambiguous Scenarios Task (AST). The present study results were analyzed using two-way repeated-measures Analysis of Variance (ANOVA) and Paired Samples t-test.

Results: The collected findings demonstrated that after receiving CBMI, children's propensity to positively interpret ambiguous situations was increased, their tendency towards negative interpretation and OCD severity was also decreased. There was no such significant change in the control group. Furthermore, the effects of CBMI was sustained at the 2-month follow-up step.

Conclusion: This study provided preliminary evidence that suggests CBMI is capable of modifying interpretation bias in children with OCD, can reduce the severity of their disorder, and works as an online intervention. This brief and inexpensive intervention could be considered as an auxiliary or standalone treatment for OCD in children.

\section{* Corresponding Author:}

Saeed Nasiry, MSc.

Address: Department of Clinical Psychology, School of Medicine, Shahid Beheshti University of Medical Sciences, Tehran, Iran

Tel: +98 (939) 8399387

E-mail:snasiry@sbmu.ac.ir 


\section{Highlights}

- CBMI successfully reduced interpretation bias in children with OCD.

- Reduction in interpretation bias resulted in decreased OCD severity.

- Online implementation of CBMI was effective in children with OCD.

\section{Plain Language Summary}

Intrusive and troubling thoughts and impulses are experienced by almost everyone; however, individuals with Obsessive-Compulsive Disorder (OCD) interpret these thoughts and impulses as more important and more threatening than they are. This condition is called interpretation bias, i.e. an essential factor in OCD. Interpretation bias can be modified using different methods which are collectively known as Cognitive Bias Modification of Interpretation (CBMI). Previous research has suggested that CBMI can reduce interpretation bias in adults with OCD and decrease the severity of their disorder; however, no study has investigated the effects of this method in children. Therefore, this study aimed to determine the effects of CBMI on children with OCD; we also examined whether an online version of this intervention can be effective for children. We delivered this online intervention to 18 children and compared them with 17 other children who received a placebo intervention. The present study results reflected that online CBMI is capable of reducing interpretation bias in children with OCD and decreasing the severity of their disorder. So many children with OCD have no access to or fail to benefit from the common interventions for this disorder. Furthermore, this new treatment is so much more brief and inexpensive and does not even require leaving the house. Thus, CBMI can be a beneficial alternative or addition to the common treatment methods for children with OCD.

\section{Introduction}

bsessive-Compulsive Disorder (OCD) is a severe and persistent mental health condition; it is recognized as the fourth most common mental health disorder, with $1.3 \%$ to $3.8 \%$ prevalence (Bradley et al., 2016; De Putter \& Koster, 2017; Williams \& Grisham, 2013). This disorder can take a chronic course if left untreated (Andersson et al., 2012). Besides, OCD is on par with schizophrenia in terms of its influence on social functioning and quality of life (Schwartzman et al., 2017). It is also the tenth main cause of disability, according to the World Health Organization (Amir, Kuckertz, Najmi, \& Conley, 2015); thus, it significantly affects public health. Despite previous assumptions, the prevalence of OCD is high among children, with an estimation of 2\%-3\% (Barrett \& Healy, 2003). Moreover, high percentages of adults with OCD (33-55\%) have reported that the onset of their disorder has been before 15 years of age (Henin \& Kendall, 1997).

Despite the high prevalence of OCD, it is underdiagnosed, and $>50 \%$ of the patients receive no treatment for it (Kohn, Saxena, Levav, \& Saraceno, 2004). Studies revealed that Cognitive Behavioral Therapy (CBT) is effective in improving OCD in adults and non-adults (Salemink, Wolters, \& de Haan, 2015); however, this treatment is not acceptable or accessible for numerous patients (Black \& Grisham, 2018). Studies demonstrated that only $5 \%-25 \%$ of patients with OCD have access to CBT (Amir et al., 2015; Andersson et al., 2012) and from those, only $40 \%-60 \%$ respond to the treatment (Habedank, Lennartz, Arslan, \& Ertle, 2017; Williams \& Grisham, 2013). Therefore, it is necessary to conduct further research for the development and assessment of new treatment methods for OCD.

Intrusive and troubling thoughts that cause anxiety and distress (Obsessions), and give rise to behaviors, i.e. repeated to reduce anxiety (Compulsions), are the characteristics of OCD (Amir, Cobb, \& Morrison, 2008; Amir, Najmi, \& Morrison, 2009); however, these intrusions also occur in healthy individuals without any psychiatric diagnosis. Studies indicated that $80 \%-99 \%$ of the healthy population experience intrusive thoughts and impulses; however, those with OCD are more troubled by these thoughts and impulses, experience them for longer periods, and experience more difficulties ignoring them (Bradley et al., 2016; Calkins, Berman, \& Wilhelm, 2013). The cognitive-behavioral model considers cognitive bias (i.e. deficits in data processing) as the cause of this difference (Cohen, Lachenmeyer, \& Springer, 2003). 
Several psychological models have been introduced to examine the etiology, maintenance, and severity of OCD, and currently, the CBT model has the most experimental support (Conley \& Wu, 2018). According to this model, the manner of interpreting and paying attention to unwanted and troublesome thoughts and impulses causes distress and leads to repetitive and ritualistic behaviors (Clayton, Richards, \& Edwards, 1999; Clerkin \& Teachman, 2011). In other words, patients with OCD, interpret intrusive impulses and thoughts as more important and more threatening than they are (interpretation bias), and pay more attention to information related to these thoughts and impulses (attention bias). In the past two decades, numerous studies have been conducted in the field of experimental psychology to examine this model. Accordingly, the results suggested a significant relationship between these cognitive biases and the severity of OCD in adults and children (Barrett \& Healy, 2003; Calleo, Hart, Björgvinsson, \& Stanley, 2010; Choi \& Lee, 2015; Cisler \& Olatunji, 2010).

Researchers have designed instruments to manipulate cognitive biases, and have evaluated the effects of these manipulations on OCD. In these novel interventions, i.e. collectively known as Cognitive Bias Modification (CBM), participants encounter a series of stimuli related to their Interpretation biases (CBMI) or Attention biases (CBMA) and learn to differently process these stimuli (Williams \& Grisham, 2013). Several studies revealed that CBMI and CBMA reduce cognitive biases in individuals with OCD. CBMA only reduces attention bias towards OCD-related stimuli, and presents no significant effect on the severity of OCD (Habedank et al., 2017; Mohsenpourian, Nokani, Jamilian, \& Ghasemi, 2019; Rouel \& Smith, 2018); while CBMI not only impacts interpretation bias, but also reduces OCD severity (Black \& Grisham, 2016; Clerkin \& Teachman, 2011; Dalir, Alipour, Zare, \& Farzad, 2016).

CBMI, in comparison with the main treatment of OCD, i.e. CBT, requires spending less time (both in terms of the number of sessions and the duration of each session) and is cost-effective. Moreover, since it is computerized, it has the option of online implementation (Clarke, Notebaert, \& MacLeod, 2014). Therefore, this intervention could be accessible as an auxiliary or standalone treatment for a large number of individuals in need of treatment. Despite these benefits, this novel intervention received inadequate testing. Specifically, almost all experimental studies in this area have been limited to adult populations. To the best of our knowledge, only one study has examined the effects of CBMI on OCD in adolescents (Salemink et al., 2015), and no studies have examined its effects in children with OCD. Thus, the present study aimed to explore the effects of CBMI on OCD in children.
Based on the research background in the field of CBM, we hypothesized that delivering CBMI to children will result in the reduction of their interpretation bias. Additionally, considering the role of cognitive biases in etiology, maintenance, and severity of OCD, our second hypothesis was that to the degree that CBMI intervention reduces children's interpretation bias, the severity of their OCD will also decrease.

\section{Methods}

The target population of this study was all the elementary school students in Tehran City, Iran. The inclusion criteria of the present study included the following: receiving the diagnosis of OCD based on the Diagnostic and Statistical Manual of Mental Disorders, $5^{\text {th }}$ Edition (DSM-5), an age range of 7-12 years, not having any severe illnesses requiring immediate treatment, and not having any learning disabilities. The exclusion criteria of the study were: receiving the diagnosis of comorbid disorders based on DSM5 and deciding not to complete the participation in the study. Convenience sampling was conducted by referring to several elementary schools in Tehran. Overall, 1074 children completed the Obsessive-Compulsive Inventory-Child Version (OCICV); of those, 229 children with scores of $\geq 15$ were identified. Accordingly, diagnostic interviews were conducted with them via telephone. Furthermore, 49 children who were diagnosed with OCD, presented no comorbid disorders or disabling illnesses. Besides, they had no learning disabilities and were selected to participate in the study. Then, the study participants were randomly assigned to the experimental and control groups. The experimental group received CBMI and the controls received placebo treatment. Additionally, 14 participants were excluded due to failure to complete their participation. Eventually, the data obtained from 35 children (19 females) were analyzed in this study; from which, 18 participants were in the experimental group. The age of research participants ranged from 7 to 12 years $(\mathrm{Mean} \pm \mathrm{SD}=9.62 \pm 1.37$ ).

The following tools were employed in this study to collect the required data.

Obsessive-Compulsive Inventory-Child Version (OCICV): To assess obsessions and compulsions in children, we used OCICV, i.e. designed by Foa et al. (2010) that assesses OCD in 7-17-year-olds. This 21item self-report questionnaire is scored on a 3-point scale, ranging from 0 to 2 (never, sometimes, always). Thus, the overall score of the measure ranges between 0 and 42. Like its adult version, OCICV includes 7 subscales of washing, checking, hoarding, doubting, order- 
ing, obsessing, and neutralizing; therefore, the full range of OCD symptoms can be assessed using this instrument (Foa, Kozak, Salkovskis, Coles, \& Amir, 1998). Cronbach's alpha coefficient was estimated to be 0.85 for the whole questionnaire, and between 0.81 and 0.88 for its subscales, which supports the internal consistency of the measure (Foa et al., 2010). Besides internal consistency, structural validity, construct validity, and test-retest reliability of this questionnaire have also been approved in various studies among clinical and non-clinical samples (Aspvall et al., 2020; Jones et al., 2013; Rodríguez-Jiménez et al., 2016). Karimi et al. (2015) verified the internal consistency, structural validity, and construct validity of the Persian version of this questionnaire.

Obsessive Beliefs QuestionnaireChild Version (OBQCV): For assessing OCD-related beliefs in children, the OBQCV (Coles et al., 2010) was used. This self-report measure contains 44 items and 3 subscales of Responsibility and Threat Estimation (RT), Perfectionism and Intolerance of Uncertainty (PC), as well as Importance and Control of Thought (ICT). The child version of this instrument was designed by a subgroup of Obsessive Compulsive Cognitions Working Group (OCCWG) in 2004 (Wolters et al., 2011). Cronbach's alpha coefficient was estimated to be 0.95 for the whole questionnaire, and between 0.81 to 0.93 for its subscales; these data support its internal consistency and the correlation of the results of this questionnaire with related instruments, which verifies its construct validity (Coles et al., 2010). Furthermore, test-retest reliability and structural validity of this measure have been approved in other studies (Schultz et al., 2018; Wolters et al., 2011). Halvaiepour, Khormaei, Khanzadeh, \& Nosratabadi (2013) examined the structural validity of the Persian version of this questionnaire, and Shojaei, Yousefi, and Chalabianioo (2018) explored its internal consistency, test-retest reliability, and construct validity; all of which were confirmed.

Ambiguous Scenarios Computerized Task (ASCT): To evaluate the research participants' interpretation bias, this tool was used, i.e. designed by Vassilopoulos, Banerjee, and Prantzalou (2009) to assess interpretation bias in socially anxious children. In this task, respondents are presented with ambiguous scenarios and requested to imagine themselves in those situations. After presenting each scenario, two thoughts, i.e. likely to come into the participant's mind are presented, one of which is negative, and the other is positive. The participant scores how likely it is for each of these thoughts to occur to them on a 5-point Likert-type scale (Orchard, Apetroaia, Clarke, \& Creswell, 2017). Instead of scenarios related to social anxiety, we used OCD-related scenarios in this study, i.e. inspired by a study conducted by Clerkin and Teachman (2011). These scenarios were designed based on the 6 domains of obsessive beliefs, i.e. defined by Obsessive Compulsive Cognitions Working Group (2001). For each measurement at pre-test, post-test, and follow-up phases, 25 scenarios (75 in total) were used. Minimum and maximum scores for positive and negative interpretations were 25 and 125 , respectively. Cronbach's alpha coefficient was estimated to be 0.77 for the total score of positive interpretation and 0.79 for the total score of negative interpretation, which highlighted the internal consistency of this task. Considering the odds of deficiency in children's reading skill, each scenario with its following thoughts were presented in visual and auditory formats.

Cognitive Bias Modification of Interpretation (CBMI): CBMI, like the ASCT, includes scenarios related to OCD in which participants should imagine themselves. After providing each scenario, two options are presented, one of which explains what negatively happens in the scenario, and the other one explains it neutrally. Respondents are requested to consider which option could be the "correct" one and select it. The neutral option is defined as correct, and the negative option is incorrect, and feedback about the participant's response is given accordingly (Orchard et al., 2017). In other words, after selecting each option, its correctness or incorrectness is (visually \& auditory) stated to the participant. Thus, during CBMI, participants learn not to negatively interpret ambiguous situations (Vassilopoulos et al., 2009).

CBMI includes 8 sessions, i.e. conducted every other day, and in each session, 25 scenarios are presented. Therefore, avoiding the negative interpretation of 200 unique scenarios was trained in 16 days. The scenarios' content was based on different areas of obsessive beliefs, i.e. defined by Obsessive Compulsive Cognitions Working Group (2001). Placebo treatment was designed to be methodologically equivalent to CBMI to control the time spent on the computer (Salemink, Kindt, Rienties, \& Van Den Hout, 2014). The only difference between CBMI and placebo treatment was that the scenarios in the placebo treatment were unrelated to OCD and the options after each scenario were comprehension tests, instead of negative and neutral interpretations.

This was a quasi-experimental research with pre-testpost-test-follow-up and a control group design. The experimental group received CBMI and the controls received placebo treatment. In the first session and before the start of the training, a pre-test was conducted which included OBQCV and ASCT. Apart from the first and last sessions 
which took longer than others (because of performing the pre-test and post-test), other sessions lasted for about 20 minutes each. Eightfold sessions were conducted every other day and their online implementation facilitated conducting each session at the most appropriate time of the day for the study participants. Reassessment was performed once after the last session as the post-test, and another time as a 2-months follow-up; both of which included OCICV, OBQCV, and ASCT. The collected results were analyzed using two-way repeated-measures Analysis of Variance (ANOVA) and Paired Samples t-test. Besides, the group differences at baseline were analyzed using Independent Samples t-tests for the continuous data and Chi-squared test for the qualitative data.

Throughout the study, all the ethical guidelines were all considered. The subject, purpose, and procedure of the study were explained to all the children and their mothers, and informed consent forms were obtained from them. Furthermore, the research participants were allowed to leave the study whenever desired. The study's protocol was per the ethical principles of the $1975 \mathrm{dec}$ laration of Helsinki. At the end of the study, the obtained results and their meaning were explained to the study participants and their mothers. Moreover, we presented our appreciation for their contribution to the study.

\section{Results}

To evaluate the differences of the study groups concerning demographic and clinical characteristics at the baseline, the related quantitative data were examined using Independent Samples t-test. Furthermore, the obtained qualitative data were analyzed using the Chisquared test. The collected results revealed no significant difference between the study groups in terms of age, gender, education, the duration of OCD symptoms, and pharmaceuticals use (Table 1).

The first hypothesis of this study was that CBMI could reduce children's interpretation bias. To test this hypothesis, two-way repeated-measures ANOVA was conducted to examine the significance of the differences between the research groups respecting interpretation bias. In this analysis, the group was defined as the betweensubjects factor, time was addressed as a within-subjects factor, and the total scores of positive and negative interpretation in the ASCT (Table 2) were considered as

Table 1. Demographic and clinical characteristics of the study groups

\begin{tabular}{|c|c|c|c|c|}
\hline Variables & Experimental Group & Control Group & Statistic & $\mathbf{P}$ \\
\hline Age $(y)($ Mean $\pm S D)$ & $9.21 \pm 1.45$ & $9.72 \pm 1.39$ & $t=1.06$ & 0.29 \\
\hline Gender (male-female) & $8-10$ & $8-9$ & $x^{2}=0.02$ & 0.87 \\
\hline Education (first period-second period) & $6-12$ & $10-7$ & $x^{2}=2.28$ & 0.13 \\
\hline $\begin{array}{l}\text { OCD symptoms duration (y) } \\
\text { (Mean } \pm \text { SD) }\end{array}$ & $2.48 \pm 1.63$ & $2.13 \pm 1.35$ & $t=0.68$ & 0.49 \\
\hline Pharmaceuticals use (yes-no) & 4-14 & $2-15$ & $x^{2}=0.67$ & 0.41 \\
\hline
\end{tabular}

Table 2. Total Mean \pm SD scores of the ASCT

\begin{tabular}{|c|c|c|c|}
\hline \multirow{2}{*}{ Group } & \multirow{2}{*}{ Time } & \multicolumn{2}{|c|}{ Mean士SD } \\
\hline & & Positive & Negative \\
\hline \multirow{3}{*}{ Experimental } & Pre-test & $73.42 \pm 11.18$ & $75.33 \pm 9.68$ \\
\hline & Post-test & $87.38 \pm 12.56$ & $67.45 \pm 10.08$ \\
\hline & Follow-up & $85.98 \pm 13.21$ & $66.87 \pm 10.23$ \\
\hline \multirow{3}{*}{ Control } & Pre-test & $79.75 \pm 16.32$ & $73.14 \pm 13.28$ \\
\hline & Post-test & $79.11 \pm 14.83$ & $71.69 \pm 11.88$ \\
\hline & Follow-up & $76.91 \pm 15.41$ & $70.99 \pm 12.31$ \\
\hline
\end{tabular}


Table 3. Results of the two-way repeated-measures ANOVA for ASCT

\begin{tabular}{|c|c|c|c|c|c|}
\hline Source & Variable & $\mathbf{F}$ & df & $\mathbf{P}$ & $n^{2}$ \\
\hline \multirow[b]{2}{*}{ Group } & Positive & 8.86 & 1.33 & $<0.001$ & 0.62 \\
\hline & Negative & 10.32 & 1.33 & $<0.001$ & 0.71 \\
\hline \multirow{2}{*}{ Time } & Positive & 7.43 & 1.33 & $<0.01$ & 0.54 \\
\hline & Negative & 8.21 & 1.33 & $<0.001$ & 0.59 \\
\hline \multirow{2}{*}{ Group $\times$ time interaction } & Positive & 11.29 & 1.33 & $<0.001$ & 0.76 \\
\hline & Negative & 10.98 & 1.33 & $<0.001$ & 0.74 \\
\hline
\end{tabular}

Table 4. Total Mean \pm SD scores of the OCICV and OBQCV

\begin{tabular}{ccccc}
\hline \multirow{2}{*}{ Groups } & Variables & & Mean \pm SD & \\
\cline { 3 - 5 } & & Pre-test & Post-test & Follow-up \\
\hline Experimental & OCICV & $16.73 \pm 7.64$ & $10.11 \pm 7.32$ & $11.74 \pm 6.81$ \\
& OBQCV & $113.34 \pm 26.59$ & $91.17 \pm 27.43$ & $89.51 \pm 29.09$ \\
Control & OCICV & $17.33 \pm 7.56$ & $16.78 \pm 7.61$ & $14.94 \pm 8.35$ \\
& OBQCV & $109.65 \pm 32.02$ & $112.91 \pm 28.58$ & $104.77 \pm 33.29$ \\
\hline
\end{tabular}

dependent variables. The two-way repeated-measures ANOVA data indicated that the effects of group, time, and the interaction of group and time have been significant on positive and negative interpretation (Table 3 ). In other words, there was a significant difference between the research groups regarding the total scores of positive interpretation and negative interpretation at multiple measurements.
After significant effects were detected in ANOVA, a Paired Samples t-test was conducted using the total scores of positive interpretation and negative interpretation of the study groups in the three measurements. This measure helped to discern the exact points of significant differences. The results of these tests revealed significant differences only in the experimental group who received CBMI; this significant difference was observed while comparing the total scores of positive interpretations at pre-test and post-test $(\mathrm{t}=3.75, \mathrm{P}<0.001)$; pre-test and

Table 5. Results of the two-way repeated-measures ANOVA for OCICV and OBQCV

\begin{tabular}{|c|c|c|c|c|c|}
\hline Sources & Variables & $\mathbf{F}$ & df & $\mathbf{P}$ & $\eta^{2}$ \\
\hline \multirow{3}{*}{ Group } & $\mathrm{OCICV}$ & 6.59 & 1.33 & $<0.01$ & 0.46 \\
\hline & & & & & \\
\hline & OBQCV & 8.32 & 1.33 & $<0.001$ & 0.60 \\
\hline \multirow{2}{*}{ Time } & $\mathrm{OCICV}$ & 5.41 & 1.33 & $<0.01$ & 0.39 \\
\hline & OBQCV & 8.94 & 1.33 & $<0.001$ & 0.63 \\
\hline \multirow{2}{*}{$\begin{array}{l}\text { Group } \times \text { time } \\
\text { interaction }\end{array}$} & $\mathrm{OCICV}$ & 7.38 & 1.33 & $<0.01$ & 0.58 \\
\hline & OBQCV & 9.43 & 1.33 & $<0.001$ & 0.66 \\
\hline
\end{tabular}


follow-up ( $\mathrm{t}=3.31, \mathrm{P}<0.01$ ); the total scores of negative interpretations at pre-test and post-test $(\mathrm{t}=2.65, \mathrm{p}=0.01)$, and pre-test and follow-up $(\mathrm{t}=2.73, \mathrm{P}<0.01)$. However, the control group who received placebo treatment indicated no significant difference neither in the total scores of positive interpretations nor in the total scores of negative interpretations at pre-test, post-test, and follow-up measurements.

The second hypothesis of the current study was that to the degree that CBMI reduces children's interpretation bias, their OCD severity would also decrease. To test this hypothesis, the difference in the total scores of OCICV and OBQCV were evaluated between the research groups at the three measurements. For this purpose, twoway repeated-measures ANOVA was used; accordingly, the group was defined as a between-subjects factor, time was considered as a within-subjects factor, and the total scores of OCICV and OBQCV (Table 4) were addressed as dependent variables. The two-way repeated-measures ANOVA results suggested that the effects of group, time, and the interaction of group and time were significant on OCICV and OBQCV (Table 5). In other words, there was a significant difference between the study groups in terms of the total scores of OCICV and OBQCV at multiple measurements.

After significant effects were revealed by ANOVA, Paired Samples t-tests were conducted using the total scores of OCICV and OBQCV of the two groups in the three measurements (to discern the exact points of significant differences). The relevant results illustrated significant differences only in the experimental group who received CBMI. Moreover, this significant difference was observed while comparing the total scores of OCICV at pre-test and post-test $(\mathrm{t}=2.81, \mathrm{P}<0.01)$; pretest and follow-up $(\mathrm{t}=2.22, \mathrm{P}=0.03)$; in the total scores of OBQCV at pre-test and post-test $(\mathrm{t}=2.64, \mathrm{P}=0.01)$, and pre-test and follow-up $(\mathrm{t}=2.76, \mathrm{P}<0.01)$. The control group participants who received placebo treatment presented no significant difference in OCD severity at pre-test, post-test, and follow-up measurements.

\section{Discussion}

The present study was the first to examine the effects of CBMI on interpretation bias and OCD severity in children with this disorder. In this study, 8 sessions of the online training of CBMI was delivered to the explored children with OCD, and its effects were compared with placebo treatment. Thus, an experimental group and a control group were included in this research.
The present study results revealed that CBMI successfully modified children's interpretation bias. This is because in the children who received CBMI, the tendency to positively interpret ambiguous situations in the ASCT was significantly increased, and the negative interpretations in this task were significantly decreased. This significant change was observed at post-test and was maintained at the 2-month follow-up. These results, which support the first hypothesis of the current study, were per prior research that indicated CBMI can modify the selective processing of information (Menne-Lothmann et al., 2014). Moreover, its online implementation was also effective (Salemink et al., 2014). Previous research has demonstrated that using the same scenarios in the assessment and the modification of interpretation bias can cause response bias and reduce the credibility of the results (Amir et al., 2015). Therefore, different scenarios were used in the assessment and modification stages of the present study; accordingly, the observed results were not caused by response bias. This is because increased positive interpretation and decreased negative interpretation of the ambiguous situations were no limited to the scenarios in the modification stage. Instead, they have transferred to new scenarios in the assessment stage.

Another study result was that CBMI reduced the severity of OCD in children. Accordingly, there was a significant decrease in the total scores of OCICV and OBQCV at post-test in the children who received CBMI. This significant decrease was maintained until the 2-month follow-up. These results supported the second hypothesis of the present study. The cognitive-behavioral model of etiology, maintenance, and severity of OCD focuses on the selective processing of information and the interpretation of intrusive thoughts and impulses (Clayton et al., 1999; Clerkin \& Teachman, 2011). The obtained data suggested that it is also applicable to children. These findings add to the growing evidence supporting CBMI as an auxiliary or standalone treatment for OCD. However, this is the first study examining the effects of this treatment in children with OCD, and the replication of its results is necessary for future studies.

The present study had some limitations. First, in addition to conducting the process of CBMI, the clinical evaluation of the study participants was performed online (except for the DSM-5 diagnostic interview, i.e. conducted via telephone) and indirectly. Therefore, the only criterion of OCD severity in the children were their reports in the self-report measures used in the study; however, assessment through behavioral tasks and examination by clinical experts could manifest a more precise and comprehensive evaluation of the severity of OCD 
in the. Thus, this procedure is recommended to be used in future studies. Another limitation of the current study was a short-term follow-up of 2 months that supported the relative reliability of CBMI. However, it is an inadequate criterion guaranteeing the long-term reliability of this novel treatment. Thus, we suggest future studies to implement longer follow-ups. Furthermore, per previous research (Beadel, Smyth, \& Teachman, 2014), to modify the cognitive biases of interpretation, we used the scenarios for all study participants and covered all domains of OCD-related beliefs defined by Obsessive Compulsive Cognitions Working Group (2001). However, considering the low possibility for all of these beliefs to be present in one person with OCD, future research is suggested to determine the belief domains of each participant and only use scenarios related to those domains to modify their cognitive biases of interpretation. Whether such unique training for each participant could increase the efficacy of CBMI or not, can be a suitable subject for further study.

\section{Conclusion}

The current study data provided preliminary evidence, demonstrating that the online delivery of computerized CBMI training can successfully modify the interpretation bias of children with OCD and reduce the severity of their disorder; these effects were relatively reliable. Considering that numerous children with OCD have no access to common treatments or fail to respond to them, CBMI, as a brief and inexpensive intervention, can be used as an auxiliary or standalone treatment for them. Further research in this area is also necessary.

\section{Ethical Considerations}

\section{Compliance with ethical guidelines}

All ethical principles are considered in this article. The participants were informed of the purpose of the research and its implementation stages. They were also assured about the confidentiality of their information and were free to leave the study whenever they wished, and if desired, the research results would be available to them.

\section{Funding}

This research did not receive any grant from funding agencies in the public, commercial, or non-profit sectors.

\section{Authors' contributions}

All authors contributed equally in preparing all parts of the research.

\section{Conflict of interest}

The authors declared no conflicts of interest.

\section{References}

Amir, N., Cobb, M., \& Morrison, A. S. (2008). Threat processing in obsessive-compulsive disorder: Evidence from a modified negative priming task. Behaviour Research and Therapy, 46(6), 728-36. [DOI:10.1016/j.brat.2008.03.001] [PMID] [PMCID]

Amir, N., Kuckertz, J. M., Najmi, S., \& Conley, S. L. (2015). Preliminary evidence for the enhancement of self-conducted exposures for OCD using cognitive bias modification. Cognitive Therapy and Research, 39(4), 424-40. [DOI:10.1007/s10608-0159675-7] [PMID] [PMCID]

Amir, N., Najmi, S., \& Morrison, A. S. (2009). Attenuation of attention bias in obsessive-compulsive disorder. Behaviour Research and Therapy, 47(2), 153-157. [DOI:10.1016/j. brat.2008.10.020] [PMID] [PMCID]

Andersson, E., Enander, J., Andrén, P., Hedman, E., Ljótsson, B. \& Hursti, T.,et al. (2012). Internet-based cognitive behaviour therapy for obsessive-compulsive disorder: A randomized controlled trial. Psychological Medicine, 42(10), 2193-203. [DOI:10.1017/S0033291712000244] [PMID] [PMCID]

Aspvall, K., Cervin, M., Andrén, P., Perrin, S., Mataix-Cols, D., \& Andersson, E. (2020). Validity and clinical utility of the obsessive compulsive inventory-child version: Further evaluation in clinical samples. BMC Psychiatry, 20(1), 42. [DOI:10.1186/ s12888-020-2450-7] [PMID] [PMCID]

Barrett, P. M., \& Healy, L. J. (2003). An examination of the cognitive processes involved in childhood obsessive-compulsive disorder. Behaviour Research and Therapy, 41(3), 285-99. [DOI:10.1016/S0005-7967(02)00011-6]

Beadel, J. R., Smyth, F. L., \& Teachman, B. A. (2014). Change processes during cognitive bias modification for obsessive compulsive beliefs. Cognitive Therapy and Research, 38(2), 103-19. [DOI:10.1007/s10608-013-9576-6]

Black, M. J., \& Grisham, J. R. (2016). Imagery versus verbal interpretive cognitive bias modification for compulsive checking. Behaviour Research and Therapy, 83, 45-52. [DOI:10.1016/j. brat.2016.05.009] [PMID]

Black, M. J., \& Grisham, J. R. (2018). A pilot study of interpretive cognitive bias modification for OCD: Targeting memory, uncertainty, and perfectionism biases. Journal of Experimental Psychopathology, 9(1), 2043808718778969. [DOI:10.1177/2043808718778969]

Bradley, M. C., Hanna, D., Wilson, P., Scott, G., Quinn, P., \& Dyer, K. F. (2016). Obsessive-compulsive symptoms and attentional bias: An eye-tracking methodology. Journal of Behavior 
Therapy and Experimental Psychiatry, 50, 303-8. [DOI:10.1016/j. jbtep.2015.10.007] [PMID]

Calkins, A. W., Berman, N. C., \& Wilhelm, S. (2013). Recent advances in research on cognition and emotion in OCD: A review. Current Psychiatry Reports, 15(5), 357. [DOI:10.1007/ s11920-013-0357-4] [PMID]

Calleo, J. S., Hart, J., Björgvinsson, T., \& Stanley, M. A. (2010). Obsessions and worry beliefs in an inpatient OCD population. Journal of Anxiety Disorders, 24(8), 903-8. [DOI:10.1016/j. janxdis.2010.06.015] [PMID]

Choi, S. A., \& Lee, J.-H. (2015). The effect of enhanced responsibility on attentional bias in obsessive-compulsive checkers. Journal of Obsessive-Compulsive and Related Disorders, 6, 27-32. [DOI:10.1016/j.jocrd.2015.04.003]

Cisler, J. M., \& Olatunji, B. O. (2010). Components of attentional biases in contamination fear: Evidence for difficulty in disengagement. Behaviour Research and Therapy, 48(1), 74-8. [DOI:10.1016/j.brat.2009.09.003] [PMID] [PMCID]

Clarke, P. J., Notebaert, L., \& MacLeod, C. (2014). Absence of evidence or evidence of absence: reflecting on therapeutic implementations of attentional bias modification. BMC Psychiatry, 14(1), 1-6. [DOI:10.1186/1471-244X-14-8] [PMID] [PMCID]

Clayton, I. C., Richards, J. C., \& Edwards, C. J. (1999). Selective attention in obsessive-compulsive disorder. Journal of Abnormal Psychology, 108(1), 171-5. [DOI:10.1037/0021-843X.108.1.171] [PMID]

Clerkin, E. M., \& Teachman, B. A. (2011). Training interpretation biases among individuals with symptoms of obsessive compulsive disorder. Journal of Behavior Therapy and Experimental Psychiatry, 42(3), 337-43. [DOI:10.1016/j.jbtep.2011.01.003] [PMID] [PMCID]

Cohen, Y., Lachenmeyer, J. R., \& Springer, C. (2003). Anxiety and selective attention in obsessive-compulsive disorder. Behaviour Research and Therapy, 41(11), 1311-23. [DOI:10.1016/S00057967(03)00037-8]

Coles, M. E., Wolters, L. H., Sochting, I., De Haan, E., Pietrefesa, A. S., \& Whiteside, S. P. (2010). Development and initial validation of the obsessive belief questionnaire-child version (OBQCV). Depression and Anxiety, 27(10), 982-91. [DOI:10.1002/ da.20702] [PMID]

Conley, S. L., \& Wu, K. D. (2018). Experimental modification of dysfunctional interpretations in individuals with contamination concerns. Journal of Behavior Therapy and Experimental Psychiatry, 59, 56-64. [DOI:10.1016/j.jbtep.2017.11.005] [PMID]

Dalir, M., Alipour, A., Zare, H., \& Farzad, V. (2016). [The effect of cognitive bias modification therapy on symptoms of obsessive-compulsive disorder: Comparison efficacy of cognitive bias modification therapy and group cognitive-behavioral therapy (Persian)]. Clinical Psychology Studies, 6(21), 1-25. [DOI:10.22054/jcps.2016.2382]

De Putter, L. M., \& Koster, E. H. (2017). The effects of obsessivecompulsive symptoms and disorder-relevant stimuli on the dynamics of selective attention. Journal of Obsessive-Compulsive and Related Disorders, 15, 74-84. [DOI:10.1016/j.jocrd.2017.09.001]

Foa, E. B., Coles, M., Huppert, J. D., Pasupuleti, R. V., Franklin, M. E., \& March, J. (2010). Development and validation of a child version of the obsessive compulsive inventory. Behavior Therapy, 41(1), 121-132. [DOI:10.1016/j.beth.2009.02.001] [PMID]
Foa, E. B., Kozak, M. J., Salkovskis, P. M., Coles, M. E., \& Amir, N. (1998). The validation of a new obsessive-compulsive disorder scale: The Obsessive-Compulsive Inventory. Psychological Assessment, 10(3), 206-14. [DOI:10.1037/1040-3590.10.3.206]

Obsessive Compulsive Cognitions Working Group. (2001) Development and initial validation of the obsessive beliefs questionnaire and the interpretation of intrusions inventory. Behaviour Research and Therapy, 39(8), 987-1006. [DOI:10.1016/ S0005-7967(00)00085-1]

Habedank, I., Lennartz, S. J., Arslan, R. C., \& Ertle, A. (2017). Online attention bias modification for Obsessive-Compulsive Disorder: A randomized controlled trial. PsyArXiv. [DOI:10.31234/osf.io/gxvwk]

Halvaiepour, Z., Khormaei, F., Khanzadeh, M., \& Nosratabadi, M. (2013). [Validation of the persian version of obsessive beliefs questionnaire-child and teenager version in group of Iranian adolescents (Persian)]. SSU_Journals, 21(3), 319-29. http:/ /jssu.ssu.ac.ir/article-1-2494-en.html

Henin, A., \& Kendall, P. C. (1997). Obsessive-Compulsive Disorder in Childhood and Adolescence. In T. H. Ollendick, \& R. J. Prinz. Advances in clinical child psychology (pp. 75-131): Springer. [DOI:10.1007/978-1-4757-9035-1_3]

Jones, A. M., De Nadai, A. S., Arnold, E. B., McGuire, J. F., Lewin, A. B., \& Murphy, T. K., et al. (2013). Psychometric properties of the obsessive compulsive inventory: Child version in children and adolescents with obsessive-compulsive disorder. Child Psychiatry \& Human Development, 44(1), 137-51. [DOI:10.1007/s10578-012-0315-0] [PMID]

Karimi, J., Homayuni Najafabadi, F., Homayuni Najafabadi, S., \& Homayuni Najafabadi, A. (2015). [Validation of a Persian version of the Obsessive Compulsive Inventory-Child Version (OCI-CV) (Persian)]. Journal of Research in Behavioural Sciences, 13(3), 388-99. http:/ / rbs.mui.ac.ir/article-1-413-en.html

Kohn, R., Saxena, S., Levav, I., \& Saraceno, B. (2004). The treatment gap in mental health care. Bulletin of the World health Organization, 82, 858-66. https://www.scielosp.org/article/ bwho/2004.v82n11/858-866/en/

Menne-Lothmann, C., Viechtbauer, W., Höhn, P., Kasanova, Z., Haller, S. P., \& Drukker, M., et al. (2014). How to boost positive interpretations? A meta-analysis of the effectiveness of cognitive bias modification for interpretation. PloS One, 9(6), e100925. [DOI:10.1371/journal.pone.0100925] [PMID] [PMCID]

Mohsenpourian, K., Nokani, M., Jamilian, H., \& Ghasemi, M. (2019). Comparing attention bias modification with selective serotonin reuptake inhibitors in patients with ObsessiveCompulsive Disorder. Journal of Arak University of Medical Sciences, 22(4), 76-85. [DOI:10.32598/JAMS.22.4.70]

Orchard, F., Apetroaia, A., Clarke, K., \& Creswell, C. (2017). Cognitive bias modification of interpretation in children with social anxiety disorder. Journal of Anxiety Disorders, 45, 1-8. [DOI:10.1016/j.janxdis.2016.10.012] [PMID] [PMCID]

Rodríguez-Jiménez, T., Piqueras, J. A., Lázaro, L., Moreno, E., Ortiz, A. G., \& Godoy, A. (2016). Metric invariance, reliability, and validity of the Child Version of the Obsessive Compulsive Inventory (OCI-CV) in community and clinical samples. Journal of Obsessive-Compulsive and Related Disorders, 9, 1-8. [DOI:10.1016/j.jocrd.2016.01.003]

Rouel, M., \& Smith, E. (2018). Attentional bias and its modification in contamination OCD symptomatology. Cognitive 
Therapy and Research, 42(5), 686-98. [DOI:10.1007/s10608-0189927-4]

Salemink, E., Kindt, M., Rienties, H., \& Van Den Hout, M. (2014). Internet-based cognitive bias modification of interpretations in patients with anxiety disorders: A randomised controlled trial. Journal of Behavior Therapy and Experimental Psychiatry, 45(1), 186-95. [DOI:10.1016/j.jbtep.2013.10.005] [PMID]

Salemink, E., Wolters, L., \& de Haan, E. (2015). Augmentation of treatment as usual with online cognitive bias modification of interpretation training in adolescents with obsessive compulsive disorder: A pilot study. Journal of Behavior Therapy and Experimental Psychiatry, 49(Part A), 112-9. [DOI:10.1016/j. jbtep.2015.02.003] [PMID]

Schultz, C., Lambek, R., Højgaard, D., Söchting, I., Thastum, M., \& Hove Thomsen, P., et al. (2018). Psychometric validation of a Danish version of the Obsessive Beliefs Questionnaire-Child Version (OBQ-CV). Nordic Journal of Psychiatry, 72(8), 621-9. [DOI:10.1080/08039488.2018.1513068] [PMID]

Schwartzman, C. M., Boisseau, C. L., Sibrava, N. J., Mancebo, M. C., Eisen, J. L., \& Rasmussen, S. A. (2017). Symptom subtype and quality of life in obsessive-compulsive disorder. Psychiatry research, 249, 307-10. [DOI:10.1016/j.psychres.2017.01.025] [PMID] [PMCID]

Shojaei, A., Yousefi, R., \& Chalabianioo, G. (2018). [Reliability and Validitymeasurement of the Obsessive Beliefs Questionnaire: Child and adolescent version (Persian)]. Journal of Clinical Psychology, 9(4), 95-105. [DOI:10.22075/jcp.2018.9192]

Vassilopoulos, S. P., Banerjee, R., \& Prantzalou, C. (2009). Experimental modification of interpretation bias in socially anxious children: Changes in interpretation, anticipated interpersonal anxiety, and social anxiety symptoms. Behaviour Research and Therapy, 47(12), 1085-9. [DOI:10.1016/j.brat.2009.07.018] [PMID]

Williams, A. D., \& Grisham, J. R. (2013). Cognitive Bias Modification (CBM) of obsessive compulsive beliefs. BMC psychiatry, 13(1), 256. [DOI:10.1186/1471-244X-13-256] [PMID] [PMCID]

Wolters, L. H., Hogendoorn, S. M., Koolstra, T., Vervoort, L., Boer, F., \& Prins, P. J., et al. (2011). Psychometric properties of a Dutch version of the Obsessive Beliefs Questionnaire-Child Version (OBQ-CV). Journal of Anxiety Disorders, 25(5), 714-21. [DOI:10.1016/j.janxdis.2011.03.008] [PMID] 